\title{
El derecho como modelo para las ciencias naturales ${ }^{1}$
}

\author{
Vittorio Villa
}

\begin{abstract}
The article begins with an exposition of the traditional manner for understanding, during centuries, the relationship between Law and natural sciences. It then describes the radical change in depth of epistemological context produced during the past four decades. Furthermore, the manner in which, within this change, law begins to appear as a model for natural sciences, by force of two diverse types of analogy between law and these sciences. The last part is dedicated to propose the author's concept regarding reasons for choosing law as a model.
\end{abstract}

\section{El modo tradicional de entender la relación entre ciencia jurídica y ciencias naturales}

En este breve ensayo deseo exponer algunas ideas propias que he desarrollado en estos últimos años, a partir del libro Teoria della scienza juridica e teoria delle scienze naturali, ${ }^{2}$ hasta llegar a los más recientes Costruttivismo e teorie del diritto $^{3}$ y Storia della filosofía del diritto analítica. ${ }^{4}$ Desde hace mucho tiempo, uno de los ámbitos principales de mi investigación es la epistemología jurídica, o,

\footnotetext{
${ }^{1}$ Agradecemos al autor y a la revista Diritto $\mathcal{E}$ Questioni pubbliche la autorización para publicar aquí la traducción del artículo "Il diritto come modello per le scienze naturali". Es la reelaboración de la comunicación presentada en el encuentro sobre "Ciencia y derecho. El juez frente a las controversias ténico-científicas" (Florencia, Italia, mayo 7-8 de 2004). Traducción de Juan José Fernández Dusso.

${ }^{2}$ Giuffrè, Milán, 1984.

${ }^{3}$ Giappichelli, Turín, 1999.

${ }^{4}$ Il Mulino, Bolonia, 2003.
} 
como podría también decirse, la teoría del conocimiento jurídico. Dentro de este ámbito de investigación, me he ocupado de manera particular de la relación entre ciencias naturales y ciencia jurídica, en el contexto del más vasto tema de la relación entre ciencias naturales y ciencias humanas.

La relación entre ciencia jurídica y ciencias naturales ha tenido en los últimos dos siglos (a partir del nacimiento de la ciencia jurídica "moderna") un comportamiento más bien constante, como lo atestigua bien el bellísimo libro de Bobbio Teoria della scienza giuridica: ${ }^{5}$ los teóricos del derecho, en el intento de mostrar que el trabajo de los juristas (y de los estudiosos del derecho en general) tenía, en algún sentido, un carácter "científico", se dedicaron reiteradamente a utilizar los modelos de cientificidad que, en cada momento, prevalecían en su propio contexto cultural. Eran modelos trasladados muy a menudo de la filosofía de las ciencias naturales (es decir, de la metaciencia natural), y por lo tanto de la reflexión metacientífica sobre las diciplinas que eran consideradas como las más avanzadas, las que habían cosechado los éxitos más importantes, o desde el punto de vista teórico, o desde el punto de vista tecnológico. En otros términos, era opinión difundida que esas diciplinas tenían mayor capacidad de obtener resultados objetivos y empíricamente controlables, de establecer "puntos de contacto neutrales y directos con la realidad".

Hay que advertir que este modelo funcionaba también "en negativo", es decir, incluso en los casos en que los estudiosos en cuestión se daban cuenta de que, naturalmente según el punto de vista reconstructivo adoptado por ellos, la ciencia jurídica no estaba en condiciones de responder a los requisitos puestos por el modelo. De hecho, este tipo de consecuencia de ninguna manera lograba incidir sobre el modelo general de cientificidad que en cada caso fuera a ser tenido como supuesto.

Un ejemplo importante en un mismo autor de ambas comportamientos, tanto el positivo como el negativo, está constituido por Ross, exponente de punta del realismo jurídico escandinavo. Este último, desde el punto de vista de la metodología descriptiva, sostiene que los juristas "por aquello que de hecho hacen en las experiencias jurídicas de tipo continental" no producen ciencia; pero que, desde el punto de vista de la metodología prescriptiva, ${ }^{6}$ podrían hacerlo, siempre y cuando, sin embargo, decidieran llevar a cabo una fuerte reconversión metodológica hacia la explícita asunción de métodos de carácter empírico.

\footnotetext{
${ }^{5}$ Giappichelli, Turín, 1950.

${ }^{6}$ Sobre la distinción entre metodología jurídica descriptiva y metodología jurídica prescriptiva, ver en particular U. Scarpelli, L'etica senza verità, Il Mulino, Bolonia, 1982, pp. 180-181.

${ }^{7}$ Ross expone estas ideas suyas en su libro más importante (A. Ross, On Law and Justice, Steven
} 
Uno de los intentos más recientes, y más importante en absoluto, de importar un modelo de cientificidad extraído de las ciencias naturales es el que ha puesto en práctica la filosofía analítica del derecho (tanto la de base normativista como la de base realista) con referencia al neopositivismo (y, más exactamente, a dos diversas versiones de esta concepción metacientífica), a partir de los años 40 del siglo pasado, primero en los países escandinavos y luego también en Italia. Es el intento puesto en práctica primero por Ross en los años 40 y 50, y después, en los años 50 y 60, por la vertiente normativista de la escuela analítica italiana (Bobbio, Scarpelli, Ferrajoli), ${ }^{8}$ sobre la base de la común aceptación del monismo metodológico (la tesis según la cual existe un solo método para todas las ciencias, el que se supone que es adoptado por los científicos naturales). ${ }^{9} \mathrm{Al}$ exponer las implicaciones de la adhesión a este supuesto común, sin embargo, los dos caminos se dividen, incluso porque son diversas las concepciones de ciencia que tienen como transfondo estos dos intentos de utilizar las ciencias naturales como modelo.

En el caso de Ross, la concepción epistemológica "de fondo" está constituida por el strict positivism, ${ }^{10}$ etiqueta que vale para representar la primera fase de desarrollo, en clave rígidamente empirista, del neopositivismo, ${ }^{11}$ en la cual se prescribe, para todas las ciencias, la adopción integral del principio de verificación. ${ }^{12}$

\& Sons, Londres, 1958; trad. it. Diritto e giustizia, Einaudi, Turín, 1990, pp. 39 ss., 46-48), en el cual sus tesis de teoría del derecho alcanzan una sistematización más madura y acabada.

${ }^{8}$ Para una reconstrucción de las tesis principales de la escuela analítica italiana, ver mi Storia della filosofia del diritto analitica, pp. 81-103.

${ }^{9}$ Para la definición de monismo metodológico ver D.L. Phillips, Wittgenstein and Scientific Knowledge. A Sociological Perspective, MacMillan, Londres, 1977, p. 59, y G.H. von Wright, Explanation and Understanding, Cornell University Press, Ithaca-New York, 1971; trad. it. Spiegazione e comprensione, Il Mulino, Bolonia, 1977, pp. 20-22.

${ }^{10}$ Uso aquí esta locución en el sentido que le atribuye J. Giedymin, Anti-positivism in Contemporary Philosophy of Science, en "British Journal of Philosophy of Science", 26,4, 1975, p. 275.

${ }^{11}$ Expresiones típicas de esta versión rígidamente empirista del neopositivismo pueden ser considerados algunos escritos de M. Schlick, Philosophical Papers, Vol. II, ed. by, H. Mulder and B.F.B. van de Welde-Schilk, Reidel, Dordrecht, 1979; de A.Y. Ayer, Language, Truth, Logic, Victor Gollancz, Londres, 1946 (first edition 1936); trad. it. Linguaggio, verià, logica, Feltrinelli, Milán, 1975 (II ed.); y finalmente, del "primer Carnap" (cfr. R. Carnap, Der logishce Aufbau der Welt, Meiner, Hamburgo, 1928; trad. it. La costruzione logica del mondo e pseudoproblemi nella filosofia, Fabbri, Milán, 1966).

${ }^{12}$ Un óptimo análisis histórico-reconstructivo del principio de verificación, en las varias fases atravesadas por él, es el de C.G. Hempel, Problems and Changes in the Empiricist Criterion of Meaning, en "Revue Internationale de Philosophie", XI, 1950; trad. it. Problemi del criterio empiristico di 
En el caso de la escuela analítica italiana, la concepción "de fondo" está constituida por el broad positivism, ${ }^{13}$ etiqueta que se puede contraponer a la anterior, y que vale para representar una segunda fase de desarrollo del neopositivismo, ${ }^{14}$ en la cual se subraya más vigorosamente el aspecto de la elaboración de un lenguaje riguroso y coherente como objetivo fundamental en la construcción de las teorías científicas ${ }^{15}$ (ver sobre todo la concepción metateórica denominada received view on theories). ${ }^{16}$

\section{El cambio radical del "contexto epistemológico de fondo"}

El cuadro epistemológico descrito arriba cambia radicalmente a partir de la segunda mitad de los años sesenta, incluso si las teorías jurídicas analíticas tardan en darse por enteradas, o bien lo hacen de manera contradictoria y matizada.

En este escrito pretendo ante todo dar cuenta, así sea en términos forzadamente muy breves y esquemáticos, de algunos importantes aspectos de este cambio, que producen, entre otras consecuencias, también la que se refiere a

significato, en Semantica e filosofia del linguaggio, por L. Linsky, Il Saggiatore, Milán, 1969, pp. 209-242. Ross adopta expresamente el principio de verificación en su Diritto e giustizia, cit., p. 39, utilizándolo como criterio fundamental para el control empírico de las hipótesis teóricas construidas por la ciencia jurídica, y marcadamente para el control de las hipótesis sobre la validez de las normas jurídicas (ibid., pp. 29-71).

${ }^{13}$ Utilizo de nuevo J. Gyedimin, Anti-positivism..., cit. P. 275, para esta segunda etiqueta.

${ }^{14}$ Expresión importante de esta segunda fase del neopositivismo pueden ser consideradas las obras del "segundo Carnap" (por ejemplo, R. Carnap, Testability and Meaning, en "Philosophy of Science", III (pp. 420-471) y IV (pp. 1-40), 1936, de Nagel (T. Nagel, The Structure of Science, Harcourt, Brace \& World, London, 1961; trad. it. La struttura della scienza, Feltrinelli, Milano, 1968) y de Braithwaite (R.B. Braithwaite, Scientific Explanation, Cambridge University Press, Cambridge, 1953; trad. it. La spiegazione scientifica, Feltrinelli, Milano, 1966).

${ }^{15}$ Desde este punto de vista es justamente famoso, incluso por su valor paradigmático, el ensayo de Norberto Bobbio, Scienza del diritto e analisi del linguaggio (1950), ahora en Diritto e analisi del linguaggio, editado por U. Scarpelli, Edizioni di Comunità, Milán, 1976, pp. 287-324. En este ensayo, Bobbio trata de mostrar cómo la dogmática jurídica puede ser considerada con todo derecho como una ciencia, así sea en sentido débil, y justamente porque se preocupa, precisamente como las ciencias naturales, por construir un lenguaje teórico riguroso, obtenido a través de una serie de operaciones — de purificación, de complemento y de sistematización — que tienen como punto de partida el lenguaje del legislador.

${ }^{16}$ Parece que ha sido Putnam el primer en servirse de esta expresión, con referencia a las concepciones metateóricas del neopositivismo de los años cincuenta (cfr. H. Putnam, Philosophical Papers, Vol. I, Cambridge University Press, Cam,bridge, 1975, p. 215). 
un modo nuevo de configurar las relaciones entre ciencia jurídica (y, más en general, "el derecho") y las ciencias naturales.

Desde la segunda mitad de los años 60 comienza a cambiar, como lo he dicho arriba, el contexto de fondo dentro del cual antes se ponía el problema de la "cientificidad de la jurisprudencia". Desde el punto de vista cuidadosamente epistemológico, comienza a entrar en una crisis profunda la imagen neopositivista de ciencia, crisis que no es ya "interna" de esta concepción, sino que anuncia un "cambio de paradigma". Sucede que el neopositivismo es sometido al ataque de muchas concepciones, que tienen como común denominador la convicción de que el neopositivismo debe ser abandonado, y que por esto pueden ser calificadas como "post-positivistas".

Virtualmente todas las principales posiciones neopositivistas son cuestionadas de hecho, y sustituidas poco a poco por posiciones alternativas. Veamos brevemente algunas de ellas, a través de una lista de temas que es necesariamente muy esquemática y resumida. ${ }^{17}$

El monismo metodológico (un solo método, empírico, que ha de ser impuesto a todas las disciplinas potencialmente científicas) es sustituido por el pluralismo metodológico (muchos métodos para muchos campos de investigación). La unidad de la ciencia es recuperada en un nivel epistemológico. ${ }^{18}$

Entra en crisis, aunque en un período más tardío, la idea según la cual la ciencia constituye la única forma, en sentido propio, de conocimiento. Esto trae como consecuencia el hecho de que también otras actividades que implican la adquisición de informaciones del mundo externo (por ejemplo, las ciencias humanas de entendimiento o comprensivas [scienze umane comprendenti] y el conocimiento de sentido común) llegan a ser progresivamente consideradas como parte del dominio del conocimiento.

\footnotetext{
${ }^{17} \mathrm{Al}$ indicar, aquí enseguida, las críticas principales que los post-positivistas dirigen contra los neopositivistas, con la eventual presentación de posiciones alternativas, sigo la pista indicada por el importante libro de M. Hesse, Revolutions and Reconstructions in the Philosophy of Science, Harvester Press, Brighton, 1980, pp. VII-XXVI. Ver también mi Teorie della scienza giuridica e teorie delle scienze naturali, cit., pp. 117-215.

${ }^{18}$ Según la feliz metáfora sugerida por Toraldo di Francia y por Piattelli Palmarini (cfr. G. Toraldo Di Francia - M. Piattelli Palmarini, Livelli della realtà, en "Problemi della transizione", II, 1979, p. 118), el discurso sobre la unidad de la ciencia pasa hoy por la construcción de "pasarelas locales, si bien provisorias, que conectan un cuerpo de conocimientos científicos con otro cuerpo de conocimientos científicos"; en este sentido, "la nueva unidad del saber se parece un poco a un archipiélago con algún transbordador irregular de isla a isla”.
} 
Es criticado el "realismo ingenuo" de fondo que presuponen muchas concepciones neopositivistas, es decir, la idea de que el lenguaje científico universal sea capaz de capturar el mundo "así como es". Se sostiene, por el contrario, que cada descripción implica un punto de vista reconstructivointerpretativo (es decir, un descarte selectivo entre lenguaje y mundo, de acuerdo con la theory-ladenness de las observaciones factuales), ${ }^{19}$ hasta el punto de que incluso las observaciones son consideradas como influenciadas por la teoría, desde el lenguaje en el cual son expresadas. Tal disposición se radicaliza después, en un período sucesivo, con la entrada en campo de las posiciones constructivistas.

El criterio del control empírico, en cualquier modo que sea configurado (como verificación, como falsación, etcétera), no sólo tiende a ser interpretado por las concepciones post-positivistas de manera siempre más laxa y débil (se pasa de la verificación a la confirmación, para llegar después a la falsación), sino que pierde también el rol absorbente y absolutamente central que al inicio le fue asignado en cuanto criterio de selección entre teorías. Cada vez más seguido se hace notar que la theory-choice puede a menudo verificarse entre alternativas teóricas en situación de sustancial "empate" respecto a las confirmaciones empíricas disponibles, o en situaciones que presentan una relación de las teorías con la experiencia que es muy débil y que envía mensajes no unívocos; en estos casos, entonces, los post-positivistas sostienen que se requieren otros criterios, de segundo orden, para escoger entre las teorías en competencia (y son criterios que remiten a características de las teorías que son distintas de las de la controlabilidad empírica, es decir, por ejemplo, la coherencia, el poder explicativo, la profundidad, y a veces hasta el valor estético y otros tipos de exigencias de carácter valorativo)..$^{20}$

Todo lo dicho hasta aquí implica el decidido rechazo, por parte de los postpositivistas, de definir la verdad, cualquier cosa que se quiera entender con esta controvertida noción, como "correspondencia con los hechos", y por lo

\footnotetext{
${ }^{19}$ Ver, por ejemplo, la formulación que de esta teoría da N.R. Hanson, Patterns of Discovery. An Inquiry into the Conceptual Foundations of Science, Cambridge University Press, Cambridge, 1958, pp. 19-30. Una buena reconstrucción de esta concepción se puede encontrar en C.R. Kordig, The Theory-Ladenness of Observation, en "Rewie of Metaphysics", 24, 1970, pp. 448-484.

${ }^{20}$ Sobre este tipo de compleja situación metateórica sigue manteniéndose insuperado el análisis de T.S. Kuhn, The Essential Tension. Selected Studies in Scientific Tradition and Change, University of Chicago Press, Chicago and London, 1977, pp. 321 ss.
} 
tanto la declarada imposibilidad, para los científicos, de salirse de cualquier esquema para ir a ver hasta qué punto la teoría en cuestión corresponde "realmente" a los datos.

Empieza a imponerse, dentro del post-positivismo, una concepción de teoría científica que es distinta de la que presentaban los neopositivistas, y que es vista ya no como estructura lógica que está en correspondencia, en cuanto a algunos puntos, con aciertos que representan la codificación lingüística de observaciones y/o de experimentos, sino más bien como producto de prácticas sociales puestas en marcha por comunidades científicas; producto interpretativo cultural e históricamente condicionado, que remite a una visión global (incluso en términos de opciones metafísicas) del campo de la experiencia en cuesión y que está necesariamente conectado a prácticas caracterizdas por la aplicación de las nociones en él contenidas.

Tiene lugar, por consecuencia, siempre dentro del campo post-positivista, una progresiva pérdida de sentido y de relevancia de la polémica contra la metafísica, polémica que había sido uno de los "caballos de batalla" de los neopositivistas. Los post-positivistas dejan de considerar el discurso metafísico en su conjunto como "completamente vaciado de significado cognoscitivo", para tenerlo más bien en cuenta como un discurso del cual eventuales resultados cognoscitivos deben ser vez por vez determinados, sin rechazos nacidos del prejuicio. Esto quiere decir que el discurso metafísico puede también revelarse "influyente" desde el punto de vista cognoscitivo, en el sentido que, por ejemplo, puede anticipar las reconstrucciones ofrecidas por las teorías científicas, indicando las coordenadas generales del trabajo de reconocimiento que después debe ser desarrollado por estas últimas dentro de un dado campo de experiencia. ${ }^{23}$

${ }^{21}$ Esta tesis es afirmada con particular claridad por N. Goodman, Ways of Worldmaking, Hackett, Indianapolis and Cambridge, 1978, pp. 2-17.

${ }^{22}$ En cuanto concierne a las críticas a la visión "axiomatizada" de las teorías científicas, propia de los neopositivistas, y la perspectiva de posiciones alternativas, que consideran a las teorías como producto histórico-cultural, ver en particular las posiciones de S. Toulmin, Human Understanding. General Introduction and Part One, Clarendon Press, Oxford, 1972, pp. 126 ss.; de L. Laudan, Progress and Its Problems. Towards a Theory of Scientific Growth, London, 1977, trad. it. Il progresso scientifico. Prospettive per una teoria, Armando, Roma, 1979, pp. 101-108; y finalmente T.S. Kuhn, The Structure of Scientific Revolutions, 2nd Edition, University of Chicago Press, Chicago, 1970, pp. 10-51.

${ }^{23}$ Constituyen buenos ejemplos de esta cambio de actitud en relación con la metafísica los trabajos de J. Agassi, Science in Flux, Reidel, Dordrecht, 1975, pp. 210 ss., 273 ss., y de J. Watkins, Metaphysics and the Advancement of Science, en "Britsh Journal for the Philosophy of Schience", 6, 1975, pp. 97 ss., y Confirmable and Influencial Metaphysics, in "Mind”, 1958, pp. 347 ss. 
Finalmente, una de las principales implicaciones de las tesis post-positivistas expuestas hasta aquí está representada por el hecho de haber sometido a discusión uno de los principios fundamentales de las concepciones tradicionales del conocimiento, es decir, del "principio de invalorabilidad" [principio di avalutatività], por lo menos en su versión más rígida, que postula la prohibición absoluta de la intromisión de los juicios de valor en el conocimiento científico. ${ }^{24}$

No se puede negar que estos cambios en la imagen general de conocimiento hayan tenido cierta influencia en el ámbito de la teoría del conocimiento jurídico, ${ }^{25}$ aunque, como lo he dicho ya, los cambios correspondientes que han tenido lugar en el ámbito jurídico se han producido con cierta lentitud, teniendo que medirse, entre otras cosas, con el impacto todavía muy fuerte de las concepciones epistemológicas tradicionales, que han terminado por mantener cierta dosis de viscosidad. De hecho, en estos últimos decenios, la concepción neopositivista ha seguido representando el punto de referencia epistemológico privilegiado y, por lo tanto, las ciencias naturales han seguido expresando su rol de modelo para la así llamada "ciencia jurídica" ${ }^{26}$ No obstante también han comenzado a desarrollar, paralelamente, algunas posturas diversas.

Por ejemplo, en el ámbito de la filosofía jurídica de orientación analítica, que constituye el campo de referencia privilegiado de mi análisis, para muchos autores el problema del "valor científico" del trabajo de los jurístas y teóricos ha comenzado a perder progresivamente peso e importancia. Se ha registrado sobre todo una neta disminución de intensidad de aquella postura obsesiva ligada a la voluntad de afirmar, a toda costa, que el trabajo de los juristas es

24 Desarrollo una crítica del principio di avalutatività, en su significado más general y en sus aplicaciones en el ámbito del conocimiento jurídico, en mi Legal Theory and Value Judgments, en "Law and Philosophy", 16, 4, 1997, pp. 447-477.

${ }^{25}$ Un óptimo ejemplo de esta influencia, en el ámbito de la filosofía jurídica italiana de los años setenta, es el trabajo de E. di Robilant, La configurazione delle teorie nella scienza giuridica, en "Rivista internazionale di filosofia del diritto", IV, 1976, pp. 470-539.

${ }_{26}$ Tomando como punto de referencia la filosofía jurídica italiana de orientación analítica contemporánea, se puede notar que ya sea la "escuela genovesa" (Guastini, Comanducci, Chiassoni, etc.) o la "escuela milanesa" (Jori, Pintore, Luzzatti, etc.) continúan reconociéndose, aun cuando en modos diversos, en la concepción neopositivista del conocimiento (científico), y por lo tanto siguen mirando a las ciencias naturales como casi paradigmáticas de discursos cumplidamente objetivos y empíricamente controlables, aunque esto no quiera decir que puedan ser considerados como modelos practicables para los discursos de los juristas; al contrario, éstos son considerados, en la mayor parte de casos, como casos paradigmáticos de discursos "políticamente y valorativamente orientados", y por lo tanto absolutamente refractarios a una inserción en la clase de los discursos científicos. 
"científico". Se ha preferido hablar, más bien, del eventual valor cognoscitivo de algunos aspectos o partes de tal trabajo.

Si vemos, por ejemplo, la producción teórica de Herbert Hart, que representa ciertamente el más importante teórico del derecho de estos últimos decenios, nos damos cuenta de que para este autor la cuestión ya no es más la de ver si el trabajo de los estudiosos del derecho (sean juristas o teóricos) es acercable al de los científicos empíricos, sino más bien la de comprobar si sus afirmaciones tienen un valor cognoscitivo, y por lo tanto alcancen a dar cuenta aceptable de algunos aspectos importantes de la experiencia jurídica.

De hecho, Hart, en el contexto de su famoso análisis dedicado a precisar la diferencia entre reglas sociales (y por lo tanto también reglas jurídicas) y hábitos, se propone el problema de cuáles son las condiciones de verdad (y no ya las condiciones de controlabilidad empírica), y por lo tanto de cuál es el valor cognoscitivo de las afirmaciones del teórico del derecho relativas a la existencia, dentro de un cierto grupo de personas, de "reglas".

\section{El derecho como modelo para las ciencias naturales}

Dentro de estos importantes cambios epistemológicos y metodológicos, contextualmente se ha verificado también otro fenómeno bastante interesante, sobre el que he puesto los ojos desde hace un tiempo. Ha sucedido que la relación entre ciencia jurídica y ciencias naturales, y, aún más en general, entre "modo de ser y de funcionar del derecho" y "modo de ser y de funcionar de la ciencia", ha dejado de tener, siempre y de todos modos, un proceder unidireccional, con las ciencias naturales sirviendo invariablemente de modelo para la ciencia jurídica y para otras varias actividades jurídicas (por ejemplo, para las de interpretación y de aplicación de normas). Es cierto que, por lo menos en algunos contextos, las ciencias naturales han seguido cumpliendo el papel de modelo (aunque interpretadas de manera distinta que en el pasado); pero ha sucedido también lo contrario: no tanto la ciencia del derecho, sino más en general, el derecho, con sus modalidades de procedimiento y sus procesos decisionales, se ha convertido en un modelo para las ciencias naturales, o, para decirlo mejor, para ciertos modos de entender y de reconstruir las ciencias naturales.

\footnotetext{
${ }^{27}$ Ver H.L.A. Hart, The Concept of Law, Second Edition with a Postscript (First Edition 1961), Clarendon Press, Oxford; trad. it. Il concetto di diritto, Torino, 2002, pp. 67-70, 105-108.
} 
Se trata de un fenómeno que tal vez puede despertar sorpresa, pero que encuentra su explicación, desde un punto de vista muy general, en el consenso cada vez más generalizado con respecto a la tesis según la cual la ciencia no tiene ya un "centro" propio, ha perdido la "disciplina piloto", aquella, en particular, considerada como la más cercana a los hechos del mundo natural, la "física". En un contexto dominado por una visión descentrada y no jerárquica de la ciencia, y desde el acercamiento del pluralismo metodológico, ninguna disciplina, ninguna concepción metodológica ligada a un contexto específico disciplinario puede atribuirse el derecho de legislar, siempre y de todos modos, incluso para las otras. Antes que el dominio de una disciplina sobre todas las otras, tiende a afirmarse en primer lugar la exploración de afinidad, de analogías pertinentes entre varios campos disciplinarios.

En este tipo de situación, mi investigación de estos últimos deceniosse ha encontrado con la posibilidad de comprobar (precisamente en el sentido de la obtención de datos, y prescindiendo, por ahora, de las opiniones que podamos tener al respecto) que casi todos los filósofos de la ciencia y los epistemólogos de inspiración en general post-positivista (Toulmin, Polanyi, Feyerabend, Kuhn, Rescher, Lakatos, Nickles, Stegmuller, etcétera) con frecuencia han mirado al derecho como modelo para las ciencias empíricas; más específicamente, han buscado en algunos fenómenos jurídicos algunos elementos para construir un modelo analógico (analogue model) para superponer a las ciencias naturales.

¿Qué cosa es un "modelo analógico"? Ahora bien, cuando se usa, ya sea en la ciencia o en la metaciencia, la locución "modelo analógico", lo que se quiere hacer es referirse a algo muy familiar, cuyas propiedades o características son más fácilmente indagables y/o comprensibles respecto a las que, consideradas en cualquier sentido similares o análogas, hacen parte del campo de fenómenos que es el objeto de la reconstrucción o de la explicación, campo de fenómenos que despierta cuestiones e interrogantes a los que no es fácil dar respuesta usando los instrumentos explicativos tradicionales. ${ }^{28}$

Una vez aclarado esto, se hace más fácil ilustrar el sentido de la hipótesis reconstructiva que presento en este trabajo. En otros términos, aquello que deseo mostrar es que la experiencia jurídica, en algunos de sus aspectos más típicos y familiares (pero también públicamente observables y de carácter institucionalizado), y más precisamente en algunas modalidades de funciona-

${ }^{28}$ La locución "modelo analógico" es usada aquí en el sentido atribuible a P. Achinstein, Concepts of Science. A Philosophical Analysis, Baltimore, 1968, pp. 208-210, 280-283. 
miento (los procedimientos que regulan los procesos jurídicos) y en algunas prácticas decisionales (las decisiones interpretativas y aplicativas de los juicios y los argumentos con los que se soportan) de nuestros sistemas jurídicos contemporáneos (modalidades y procedimientos que son representados por los filósofos de la ciencia de manera muy estandarizada y simplificada y por lo tanto sin el auxilio de específicas mediaciones teóricas), es utilizada por los filósofos de la ciencia y por los epistemólogos post-positivistas como modelo en clave heurística, con el fin de comprender más claramente algunas modalidades de funcionamiento y algunas prácticas decisionales de las ciencias naturales, que son consideradas similares de manera relevante a las precedentes. Esto quiere decir que de la experiencia jurídica son deducidas algunas representaciones esquemáticas de ciertas modalidades de decisión, o bien de ciertas modalidades de funcionamiento del sistema procesal, modalidades que sirven para comprender mejor (por ejemplo para sugerir nuevas direcciones de investigación, o bien para favorecer el desarrollo de una nueva teoría, o bien, incluso, para proveer útiles esquemas de representación) algunas modalidades de la actividad científica, en el ámbito de las ciencias naturales, modalidades que son consideradas similares, de alguna manera, a las primeras y que presentan seria dificultad en relación con su reconstrucción metacientífica.

\section{Dos tipos diversos de analogía entre derecho y ciencias naturales}

En este parágrafo distinguiré dos tipos distintos de acercamientos analógicos, dos modos distintos en los que el derecho puede funcionar como analogue model. En el parágrafo próximo y en el último, en cambio, me detendré brevemente sobre las razones que justifican la institución de estas analogías.

El primer tipo de analogía considera, de una parte, los sistemas teóricos de la ciencia; de otra parte, el sistema jurídico, según la representación simplificada de algunas de sus caracterísiticas más familiares y típicas. Las analogías consideran en particular las modalidades de funcionamiento de dos sistemas, y marcadamente el modo como "entran en relación con los hechos".

¿Qué cosas sirven para aclarar estas analogías? Sirven esencialmente para ofrecer soporte adicional y justificación a la tesis de la theory-ladenness de las observaciones, según la cual no puede existir algún tipo de lenguaje de observación "puro", es decir, autónomo e independiente de cada presupuesto o categoría teórica (el significado de las observaciones es siempre determinado por el background de conocimientos teóricos y de expectativas que los "científicos-observadores" tengan). Para este propósito se utiliza, en clave analógica, la manera en que, en ámbito jurídico, son tratadas las cuestiones de hecho 
dentro del sistema procesal. En particular se hace notar que, en tal contexto, los jueces se sirven de reglas para seleccionar los hechos que son relevantes para la decisión. Se desea en sustancia mostrar que, sea en el derecho o en la ciencia, los hechos no entran nunca en contacto con sus "sistemas de referencia" (el sistema jurídico y el sistema teórico) cuando están en su supuesto "estado puro"; se trata, por el contrario, de hechos que han recibido ya ciertas calificaciones por cuenta de normas jurídicas (en el caso del derecho) o bien por cuenta de categorias teóricas (en el caso de la ciencia).

Kuhn, por ejemplo, desarrolla este tipo de acercamiento analógico cuando hace notar que el científico, en su actividad ordinaria de "solución de problemas empíricos" antepone siempre la teoría corriente como "regla de juego". La situación sería comparable, siempre según Kuhn, a la del juez que opera dentro de un sistema de normas: en ambos casos la presencia del sistema es necesaria para dar una orientación y una "dirección metodológica de marcha" a las operaciones puestas en práctica dentro de los dos sistemas, actividades que preludian las decisiones teóricas (en el caso de los científicos) y las decisiones judiciales (en el caso de los jueces). ${ }^{29}$

Un acercamiento del mismo tipo es el que hace Lakatos, con particular referencia, en clave analógica, a los mecanismos de funcionamiento de los sistemas jurídicos de case law. El objetivo de Lakatos es el de resaltar la importancia y el valor autoritativo de las decisiones tomadas por la "elite de los científicos" en relación con las aplicaciones de determinados criterios metodológicos. Pues bien, para aclarar mejor esta situación, Lakatos compara estas decisiones con aquellas que tienen valor de precedente, puestas en práctica por los jueces en un sistema de case law. Por el contrario, los criterios propuestos en nivel de metodología (prescriptiva) son considerados similares a las prescripciones que provienen de la statute law. Aquí Lakatos quiere mostrar que así como en un sistema de case law, las normas jurídicas codificadas entran en función ante la ausencia de un precedente jurídico confiable, del mismo modo, en la ciencia, los estándares sugeridos por la metodología entran en función ante la ausencia de una práctica científica dotada de suficientes garantias de prestigiosa autoridad.

${ }^{29}$ T.S. Kuhn, Logic of Discovery or Psychology of Research ?, en Criticism and Growth of Knowledge, ed. de I. Lakatos and A. Musgrave, Cambridge University Press, Cambridge, 1979, pp. 4 ss. Consideraciones similares hacen Rescher (cfr. N. Rescher, Methodological Pragmatism, Oxford University Press, Oxford, 1977, p. 208) y Polanyi (M. Polanyi, Personal Knowledge, University of Chicago Press, Chicago, 1978, 1958'1 p. 277).

${ }^{30}$ I. Lakatos, Popper on Demarcation and Induction, en The Philosophy of Karl Popper, ed. por P.A. Schilpp, La Salle, 1974, p. 252. También Toulmin desarrolla consideraciones análogas, usando como punto de referencia analógico el sistema de case law (S. Toulmin, Human Undersatnding, cit., p. 257). 
El segundo tipo de analogía considera las elecciones y las decisiones que se producen en ambos campos. Una parte relevante de la metaciencia contemporánea está alineada a favor de la underdetermination thesis, y por lo tanto está convencida de que la theory-choice de los científicos no pueda ya, por lo menos en una serie significativa de casos, ser unívocamente justificada sobre la base de la referencia a una aplicación automática de reglas lógicas atemporales. Se hace notar, a propósito, cómo las elecciones de los científicos, en el caso de teorías en competencia, no estén muy a menudo dictadas por criterios lógicos ni de controlabilidad empírica, sino que envuelven valoraciones comparativas que remiten a criterios distintos del criterio de la confirmación empírica y del criterio de la coherencia lógica, y ulteriores a ellos. Tales decisiones no expresan, en algunos casos, sino una preferencia del científico a favor de una teoría, preferencia en apoyo de la cual él puede ofrecer tan sólo algunas "buenas razones".

Pues bien, para dar cuenta de manera más clara de tales situaciones complejas, la metaciencia natural hace referencia analógica a campos decisionales en donde son puestas en práctica selecciones que se fundan, también ellas, sobre "buenas razones" (en estos casos, sobre los valores y sobre los principios que están como fundamento, según cierta propuesta interpretativa, de la disciplina de un dado sector del derecho positivo). En fin, algunos filósofos post-positivistas están convencidos de que en los casos de theory-choice la metaciencia debería utilizar una concepción alternativa a aquella que encuentra en la lógica formal la fuente de todos los criterios; es decir, una concepción alternativa que haga referencia a los casos en los que, en ámbito jurídico (y en el ámbito más general de las decisiones prácticas) se apliquen máximas generales a casos particulares, tratando de argumentar, según modalidades alternativas a aquellas ofrecidas por la lógica formal, la mayor plausibilidad de una cierta elección (por ejemplo, de una cierta intrpretación de un principio, considerado como más relevante, en detrimento de otro en competencia con el primero).

Toulmin, por ejemplo, considera que dentro de las ciencias naturales, en ciertos momentos delicados de su historia, cobraría fuerza la exigencia de tomar decisiones científicas fundamentales, que estén en grado de reorientar, desde el punto de vista teórico, los campos disciplinarios de competencia. Según Toulmin, habría, en estas situaciones, una gran incertidumbre sobre los desarrollos futuros de esa dada disciplina, desarrollos que, al fin de cuentas, estarían determinados por elecciones metodológicas y teóricas hechas sobre la base de criterios propuestos como alternativa, y que apuntan hacia direcciones diversas; y ello daría naturalmente mucho más espacio a decisiones y elecciones estratégicas no vinculadas desde el punto de vista lógico, sino más 
bien usadas en préstamo sobre la base de criterios de razonabilidad, vueltos hacia la mayor o menor deseabilidad o preferibilidad de los varios objetos y de los varios resultados que llegarían a producirse, dentro de aquella disciplina, sobre la base de las elecciones tomadas. Ahora bien, para aclarar mejor este punto muy delicado, Toulmin encuentra útil comparar estas decisiones estratégicas de los científicos naturales con las que llegan a producirse, en ámbito jurídico, a propósito de las interpretaciones de principios constitucionales (por ejemplo, de parte de una Corte Constitucional), en un contexto en el que un único principio puede ser interpretado de modos diversos, determinando por consiguiente modos diferentes de entender la entera disciplina (en términos de reglas) de un determinado sector; o bien un conflicto entre principios en competencia (por ejemplo entre principio de igualdad y principios que sancionan derechos de libertad) puede ser resuelto de modos diversos, con consecuencias relevantes para el entero sistema de garantías de los derechos, individuales y sociales.

En ambos tipos de casos (en la ciencia y en el derecho), según Toulmin, el hecho de que las decisiones en cuestión no tengan un resultado unívocamente determinable sobre bases lógicas no debilita para nada "la naturaleza fundamentalmente racional (en términos de razonabilidad) de tales decisiones".

\section{Las razones de la elección del derecho como modelo}

Es importante ahora, en este parágrafo conclusivo, interrogarse sobre las razones de fondo que están detrás de este cambio radical en la relación entre ciencias naturales y ciencia jurídica/derecho, cambio que determina un "encaminamiento inverso" en la dirección de marcha de la relación. A propósito de esto es necesario ante todo recordar que también el neopositivismo se servía de modelos para reconstruir mejor las prácticas metodológicas de los científicos; sin embargo, se trataba prevalentemente de modelos provistos por la lógica y la matemática. Se miraba a la actividad teórica de los científicos con los lentes provistos por modelos de carácter lógico (el cálculo de las probabilidades - la lógica formal deductiva - la lógica inductiva). Estos modelos expresaban para el neopositivismo una verdadera y propia concepción de la racionalidad científica.

La crisis de las tradiciones de investigación neopositivistas termina, como es obvio, por poner también en cuestión esta concepción de la racionalidad científica. En este sentido comienza a vacilar la idea según la cual la racionalidad científica pueda ser acercada de modo exclusivo al modelo de la racionalidad lógica. No es difícil comprender, entonces, cómo la crisis de este modelo 
esté acompañada por la investigación de nuevos modelos de racionalidad científica, que estén sobre todo en grado de justificar la racionalidad (así sea en el sentido más débil de "razonabilidad") de decisiones y de procedimientos científicos no vinculados unívocamente por un sólo grupo de criterios, y que entonces son tomados en "condiciones de incertidumbre", y en un contexto que presenta criterios alternativos de elección, con todo lo que esto implica para el consiguiente pluralismo de finalidades y de resultados alcanzables de manera abstracta a través de tales elecciones.

Las frecuentes referencias al derecho, en términos de analogue model (y los ejemplos que podrían ofrecerse son de veras muchísimos, hasta llegar a filósofos contemporáneos de primer plano, como Putnam) tienen por lo tanto motivaciones muy fuertes, al estar conectadas con la investigación de nuevos modelos de racionalidad que son sin duda más débiles y elásticos, dentro de los cuales se imponen técnicas de razonamiento que justifican elecciones no unívocas, tomadas en situaciones de incertidumbre, es decir, donde a menudo está presente un abanico de argumentos, que apuntan hacia direcciones diversas.

No es difícil mostrar, y ésta es la parte menos interesante del discurso en cuanto banal y resabida, que situaciones de ese tipo son el pan de cada día de los estudios de teoría del razonamiento jurídico (en las dos vertientes de la teoría de la interpretación y de la teoría de la argumentación). En este ámbito de estudio está particularmente difundida y consolidada la tesis según la cual la justificación de las elecciones interpretativas (y por tanto también de la premisa mayor del silogismo con el que puede ser lógicamente representada la actividad de aplicación del derecho que tiene como su conclusión la sentencia) no puede ser ulteriormente fundada sobre bases lógicas, sino que es fruto, en el mejor de los casos, de "elecciones razonables", basadas en argumentos que a menudo están en competencia entre sí y que son siempre potencialmente superables por argumentos tenidos por "más fuertes".

No me detengo en este punto, en cuanto puedo darlo por descontado en un contexto de tan eminentes estudiosos del derecho. No se puede no subrayar, sin embargo, la importancia de estas constantes referencias al derecho por parte de la metaciencia contemporánea. Eso significa, entre otras cosas, que incluso en las ciencias empíricas entran en juego procedimientos de justificación racional de elecciones y de decisiones teóricas que son consideradas similares a aquellas que son puestas en práctica en el campo de la razón práctica (a propósito de esto, frecuentes son las referencias analógicas que se refieren también al campo de la moral); y esto porque se considera que, en ambos 
casos, se está en la situación de tener que justificar una elección (teórica o práctica), en presencia de una pluralidad de criterios que podrían conducir a direcciones opuestas.

Estando así las cosas, se puede entonces arrojar más de una sombra de duda sobre la concepción, bastante consolidada tanto en ámbito epistemológico como en ámbito jurídico, de que haya una oposición dicotómica entre los procedimientos de justificación práctica (en el ámbito ético-jurídico) con la que se proveen "buenas razones" para sustentar acciones, decisiones, normas, etcétera; y los procedimientos de justificación teórica, con los que (en ámbito de las ciencias empíricas) se proveen -en las situaciones de theory choice y en un contexto que acepta la underdetermination thesis- "buenas razones" a favor de una teoría, o de una hipótesis científica, tal vez en detrimento de otra.

La verdad es que, si se acepta la perspectiva post-positivista, entonces es necesario admitir que también en las ciencias empíricas entran en juego procedimientos de justificación racional de elección y de decisiones teóricas que no parecen demasiado diversas de aquellas puestas en práctica en el ámbito de las disciplinas que utilizan técnicas de razonamiento típicas de la razón práctica.

Sobre la base de este tipo de consideraciones se puede al menos notar que existe un "aire de familia", o mejor una red de "semejanzas de familia" entre justificación teórica y justificación práctica. Pero se podría tal vez sostener una tesis mucho más fuerte y afirmar por lo tanto que los procedimientos de justificación teórica, así como son configurados en ámbito post-positivista, vendrían a caer directamente dentro de las técnicas de justificación de competencia de la razón práctica.

Estas afirmaciones abren problemas filosóficos de no poca importancia, en relación sobre todo con la posible reconfiguración de las relaciones entre razón teórica y razón práctica. Se trata de problemas en los cuales no puedo ciertamente entrar, en el espacio de esta comunicación. Aquí puedo sólo hacer notar, a manera de conclusión, que el debilitamiento de la oposición "razón teórica/razón práctica" es una operación perfectamente coherente para quien, moviéndose desde premisas epistemológicas post-positivistas (o más específicamente, como en mi caso, desde premisas constructivistas), considere que también la ciencia es configurable como una práctica social guiada por reglas, y que por lo tanto, en su interior, los problemas principales son, así como para las otras prácticas sociales normativas (por ejemplo para las prácticas jurídicas), consisten en individualizar los criterios para justificar actitudes, acciones y decisiones. 